\title{
REDUÇÃO DE RUÍDOS EM SINAIS DE VIBRAÇÕES DE IMPLOSÃO DE BOLHAS NA CAVITAÇÃO EM GRANDES DUTOS DE ÁGUA
}

\section{NOISE REDUCTION IN SIGNALS OF VIBRATIONS FROM IMPLOSION BUBBLES DURING CAVITATION IN WATER DUCTS}

Fernanda Paula Barbosa Pola, Ives Renê Venturini Pola

Universidade Estadual Paulista "Júlio de Mesquita Filho" (FCT/UNESP), Faculdade de Ciências e Tecnologia, Presidente Prudente, SP.

E- mail: fernandapaulab, ivesrene)@gmail.com.

RESUMO - Esse trabalho apresenta uma análise matemática dos sinais de vibrações causadas pela implosão de bolhas de vapor d'agua presentes no escoamento em grandes dutos de água. Deste modo, o objetivo deste estudo foi identificar a duração e a frequência destas vibrações, que são importantes para estudos sobre o fenômeno de implosão de bolhas pelas suas consequências. A metodologia constou de técnicas de análise de sinais como a medição do sinal original por transformadas de Fourier e do espectro de potência para distinguir frequências dos pulsos de picos de ruídos. Assim, foi usado o espectrograma do sinal para definir a localização temporal, utilizandose outros parâmetros, de forma a aumentar sua resolução temporal. Para a determinação da localização temporal dos pulsos foi construído um filtro passa-baixa em torno de cada uma das frequências determinadas, na transformada de Fourier do sinal. Concluímos que somando todas as componentes filtradas do sinal obtemos uma aproximação do sinal original sem ruídos.

Palavras-chave: Transformada de Fourier; Análise de Sinais; Escoamentos em Dutos; Implosão de Bolhas de Vapor de Água; Redução de Ruído.

ABSTRACT - This paper presents a mathematical analysis of the signal waves of the vibration caused by the implosion of water bubbles during the fluid flow in water ducts of greater size. Therefore, the main purpose of this study was to identify the duration and the frequency of such vibrations, which are important for the study of the phenomenon of bubble implosion due to its consequences. The methodology consisted of using signal analysis techniques like the analysis of the original signal by Fourier transform and the power spectrum to distinguish the frequency of the pulses of noise peaks. The signal

Recebido em: 30/08/2016 Revisado em: 09/09/2016 Aprovado em: 19/09/2016 spectrogram helped to define the temporal location, by using different parameters, raising its temporal resolution. For the temporal determination of pulses it was used a low-pass filter around every determined frequency in Fourier transform. We concluded that 
summing up all signal filtered components resulted in an approximation of the original signal without noises.

Keywords: Fourier Transform; Signal Analysis; Duct Fluid Flow; Implosion of Water Bubbles; Noise Reduction. 


\section{INTRODUÇÃO}

O contexto deste trabalho se insere na etapa de análise de cavitação em grandes dutos de água. Especificamente, a análise se refere à identificação de vibrações características da "implosão" das bolhas de vapor d'água presentes no escoamento, através do processamento de sinais digitais [1]. Alguns autores utilizaram lógica fuzzy para a redução de ruídos em sinais digitais [2].

Estas bolhas são formadas em regiões onde ocorre uma grande perda de carga localizada, onde a pressão cai abaixo da pressão de vapor da água. Quando estas bolhas são arrastadas para outras regiões do escoamento, onde a pressão é novamente alta (acima da pressão de vapor da água), o vapor se condensa novamente, causando o colapso da bolha. Neste processo ocorrem choques entre as frentes de líquido que circundavam a bolha, cuja repetição continuada acaba por erodir as paredes da tubulação, e que geram vibrações que se propagam pelo líquido e pela tubulação.

O objetivo deste trabalho foi a identificação destas vibrações, bem como suas durações e frequências, dados importantes para estudos mais profundos do fenômeno de implosão das bolhas, seus modos e consequências.

\section{METODOLOGIA}

Primeiramente, foi realizada uma análise inicial do sinal em um gráfico, o que mostrou seu alto nível de ruído, sem nenhuma informação aparente a respeito da localização temporal e/ou frequência de qualquer pulso sonoro. $\mathrm{O}$ sinal pode ser visualizado na figura 1.

O próximo passo foi então gerar a transformada de Fourier do sinal, e determinar sua magnitude em função da frequência. O gráfico mostra apenas as frequências entre 0 e $1000 \mathrm{~Hz}$ (não redundantes). Esta é a frequência de Nyquist, a metade da frequência de amostragem, $2000 \mathrm{~Hz}$. Este gráfico é mostrado na figura 2 .

Podemos observar alguns picos significativos em frequências abaixo de $350 \mathrm{~Hz}$, mas há outros em frequências mais elevadas. Portanto, ainda é difícil distinguir frequências correspondentes a pulos sonoros de picos de ruído presentes no espectro. 


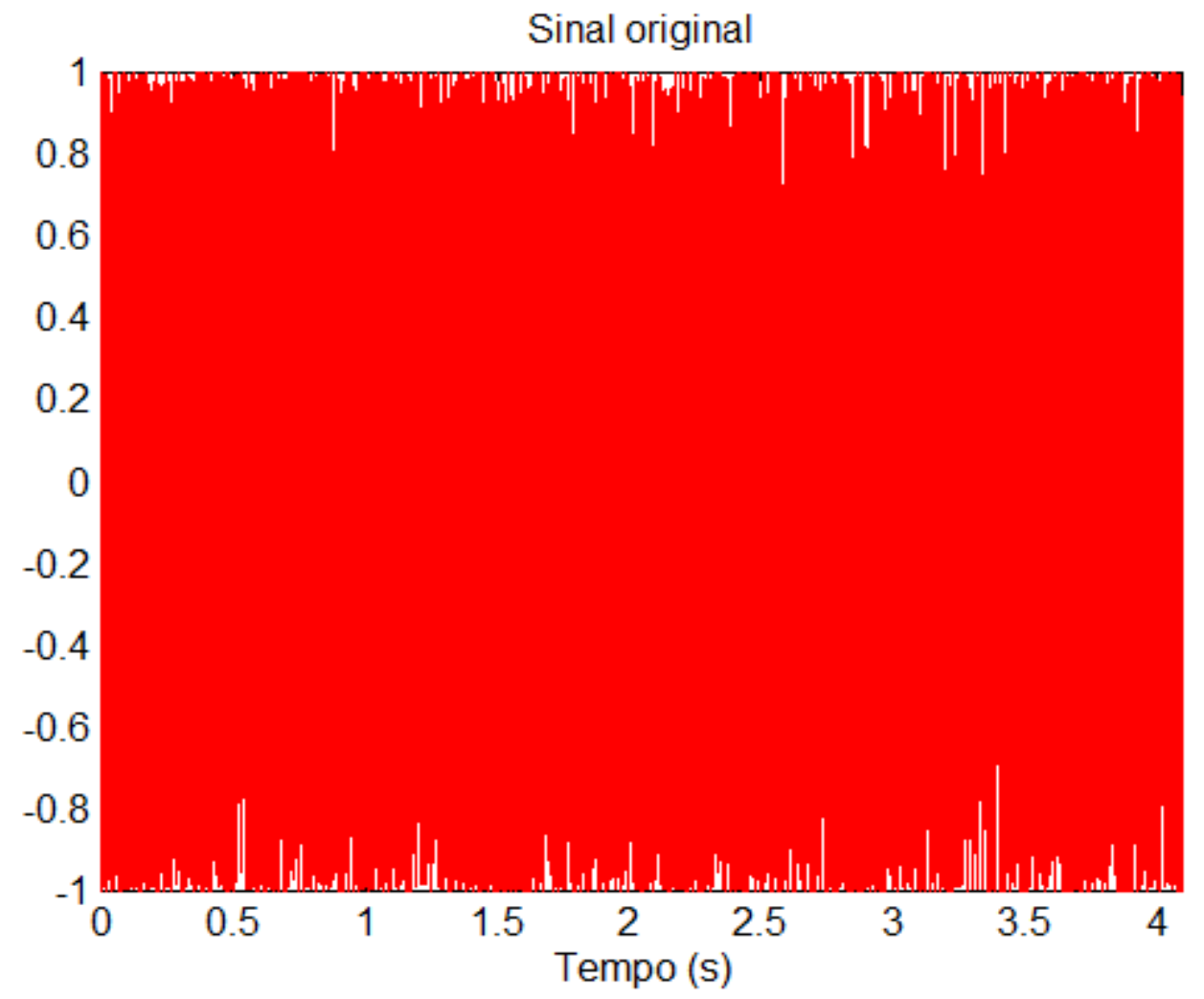

Figura 1. Representação temporal do sinal original

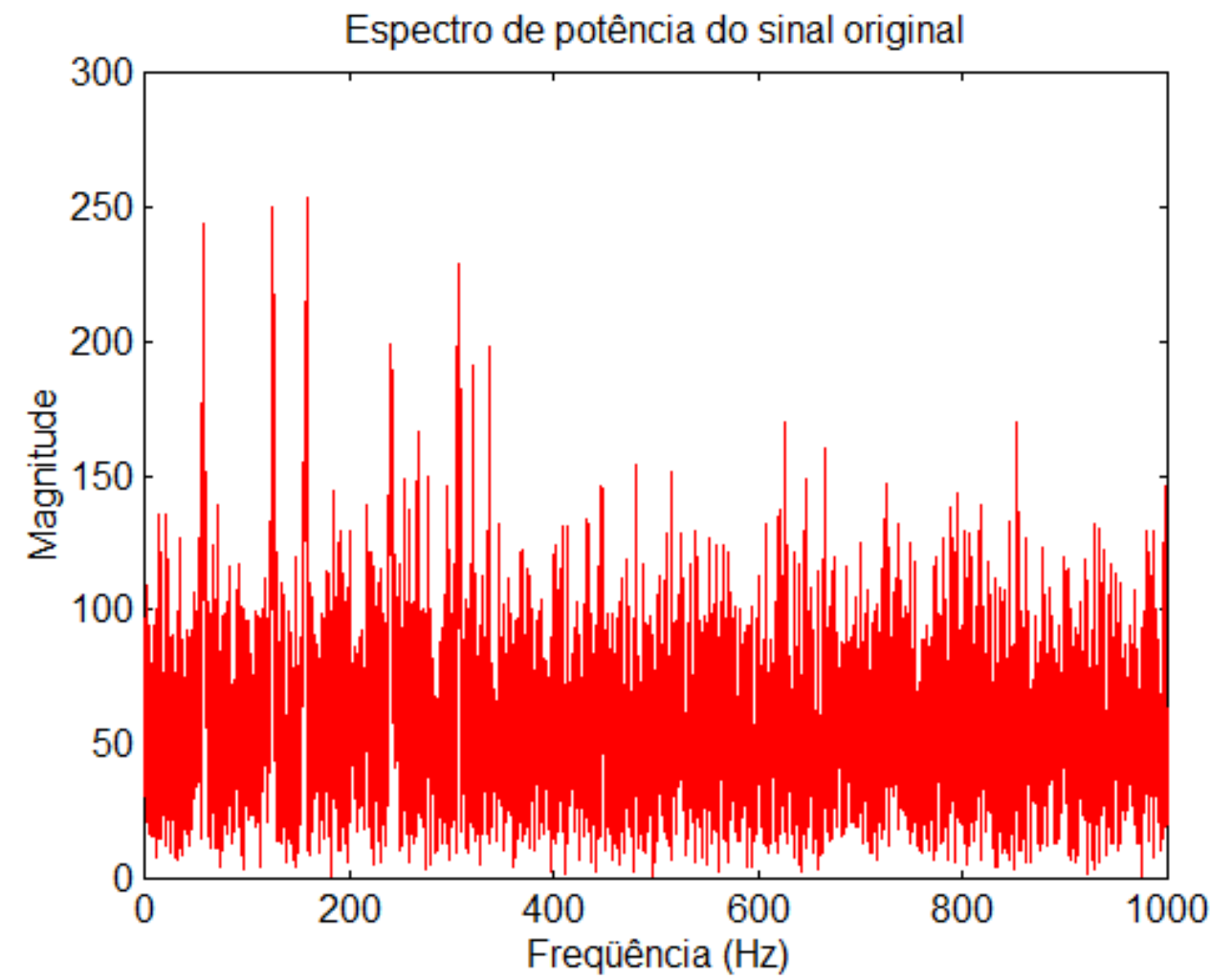

Figura 2. Representação frequencial do sinal original 
Por fim, deve ser acrescentadas as palavras chaves em português e inglês com fonte tamanho 12. As palavras "palavrachave" e "keywords" deve estar na cor azul escuro.

\section{RESULTADOS}

Para resolver este problema, ajudando a identificar as frequências dos pulsos sonoros, bem como sua localização temporal, foi gerado o espectrograma do sinal, com os parâmetros pré-definidos do Matlab $^{\circledR}$ (256 pontos para FFT, janela Hanning de 256 pontos e sobreposição de 128 pontos). Assim foram facilmente identificados 5 pulsos sonoros, como mostrado na figura 3.

Outro modo de se distinguir frequências dos pulsos de picos de ruídos é através do gráfico da função densidade de potência espectral do sinal, onde se observa claramente que apenas 5 frequências se destacam, como mostra a figura 4.

Para se determinar com maior precisão as frequências dos pulsos (já que a função densidade de potência espectral não apresenta uma boa resolução frequência) utilizou-se o recurso de "zoom" na transformada de Fourier do sinal (figura 2). Um primeiro "zoom" evidenciou a distinção dos picos de ruído das frequências presentes no sinal, como mostra a figura 5 . Outras aproximações localizadas nas frequências identificadas permitiram uma determinação mais precisa das mesmas, obtendo-se aproximadamente: $58 \mathrm{~Hz}, 125 \mathrm{~Hz}, 157 \mathrm{~Hz}$, $241 \mathrm{~Hz}$ e $307 \mathrm{~Hz}$.

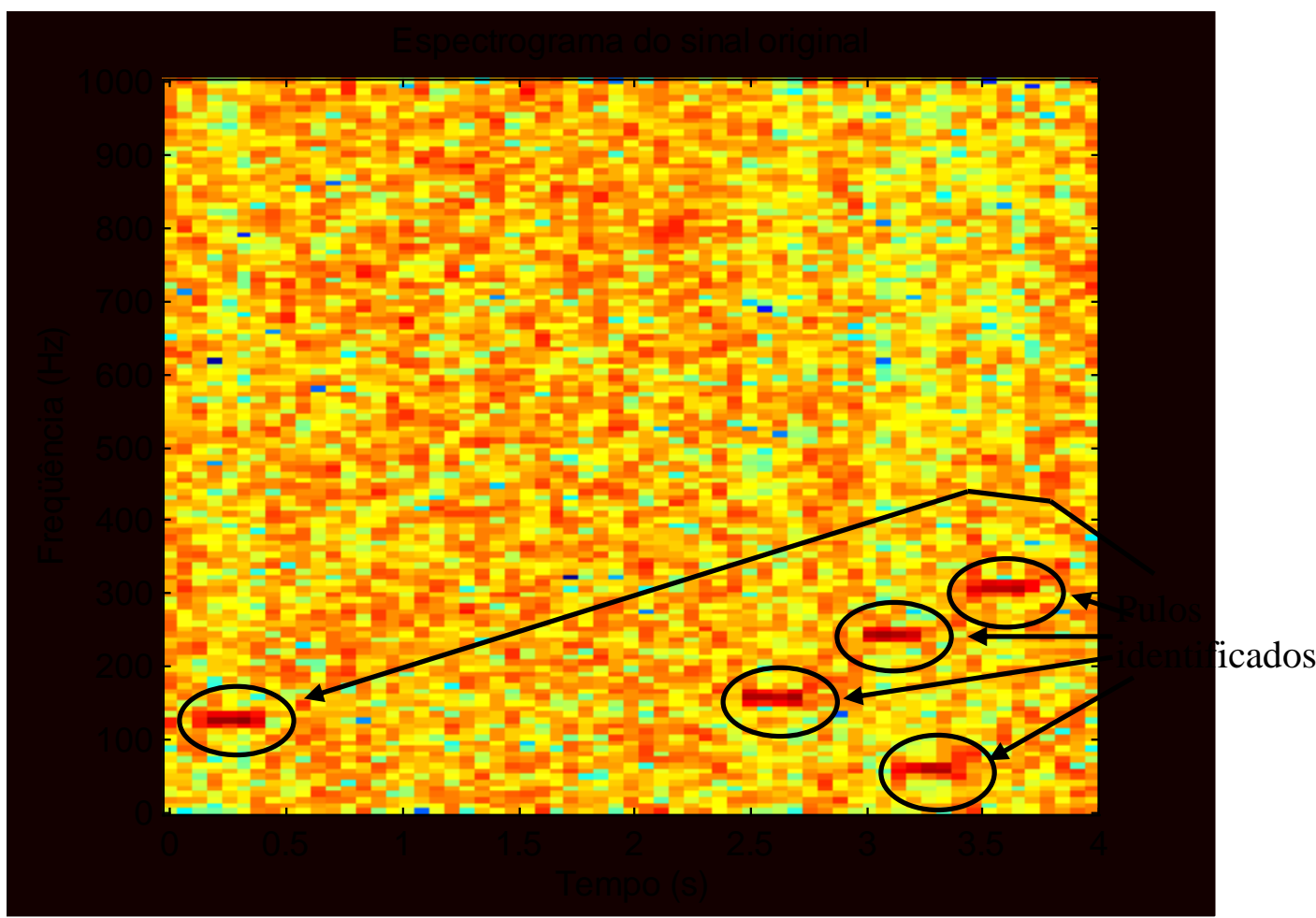

Figura 3. Representação tempo-frequencial do sinal original 


\section{DISCUSSÃO}

Para a determinação da localização temporal dos pulsos foi construído um filtro passa-baixa em torno de cada uma das frequências determinadas, na transformada de Fourier do sinal. Então tomou-se a parte real da transformada inversa de Fourier do resultado da filtragem como sendo cada componente do sinal nas frequências identificadas. Entretanto, estes gráficos não informam precisamente o início e fim dos pulsos (vide figuras 7, 8, 9, 10 e 11), devido à presença de ruído no sinal original (que apresenta componentes em todas as frequências), e devido ao fato de o filtro distorcer o sinal, uma vez que atrasa diferentemente cada componente frequência do sinal.

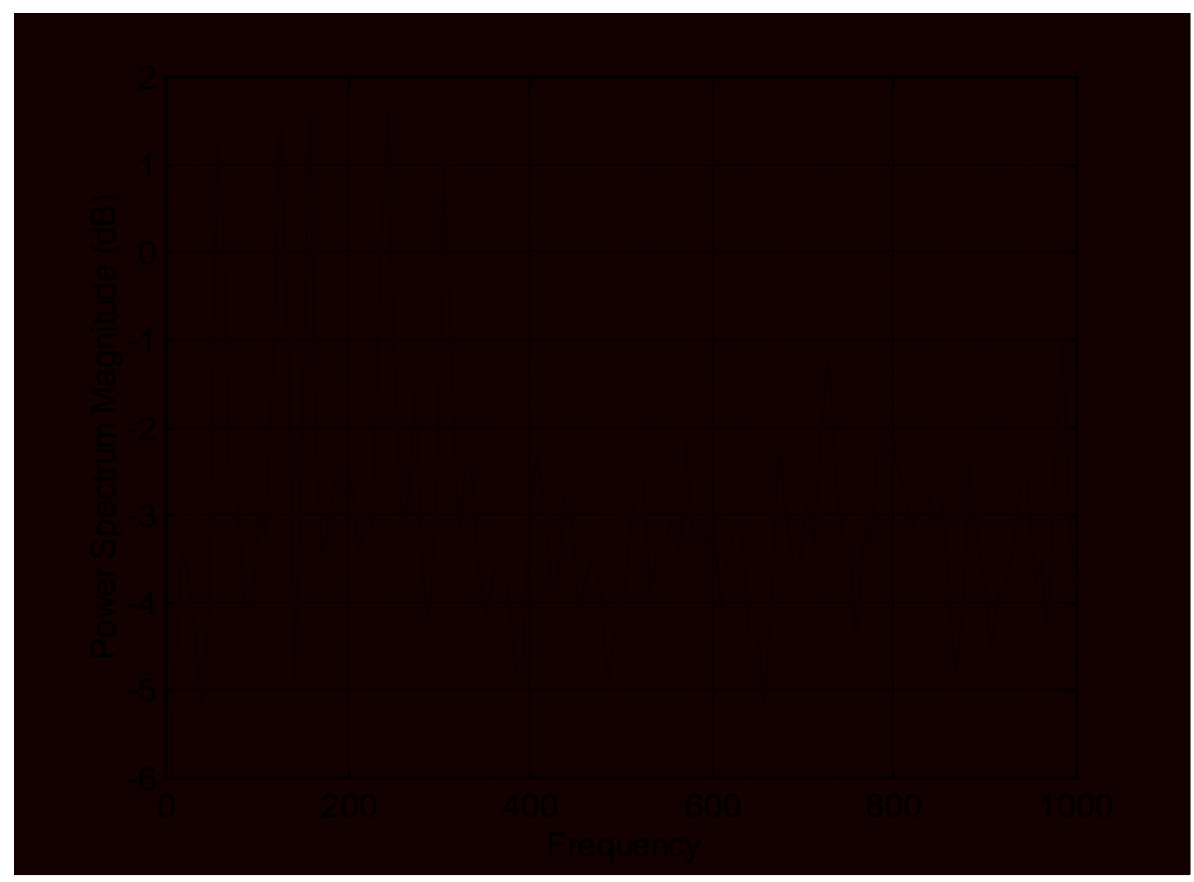

Figura 4. Densidade de potência espectral do sinal original 


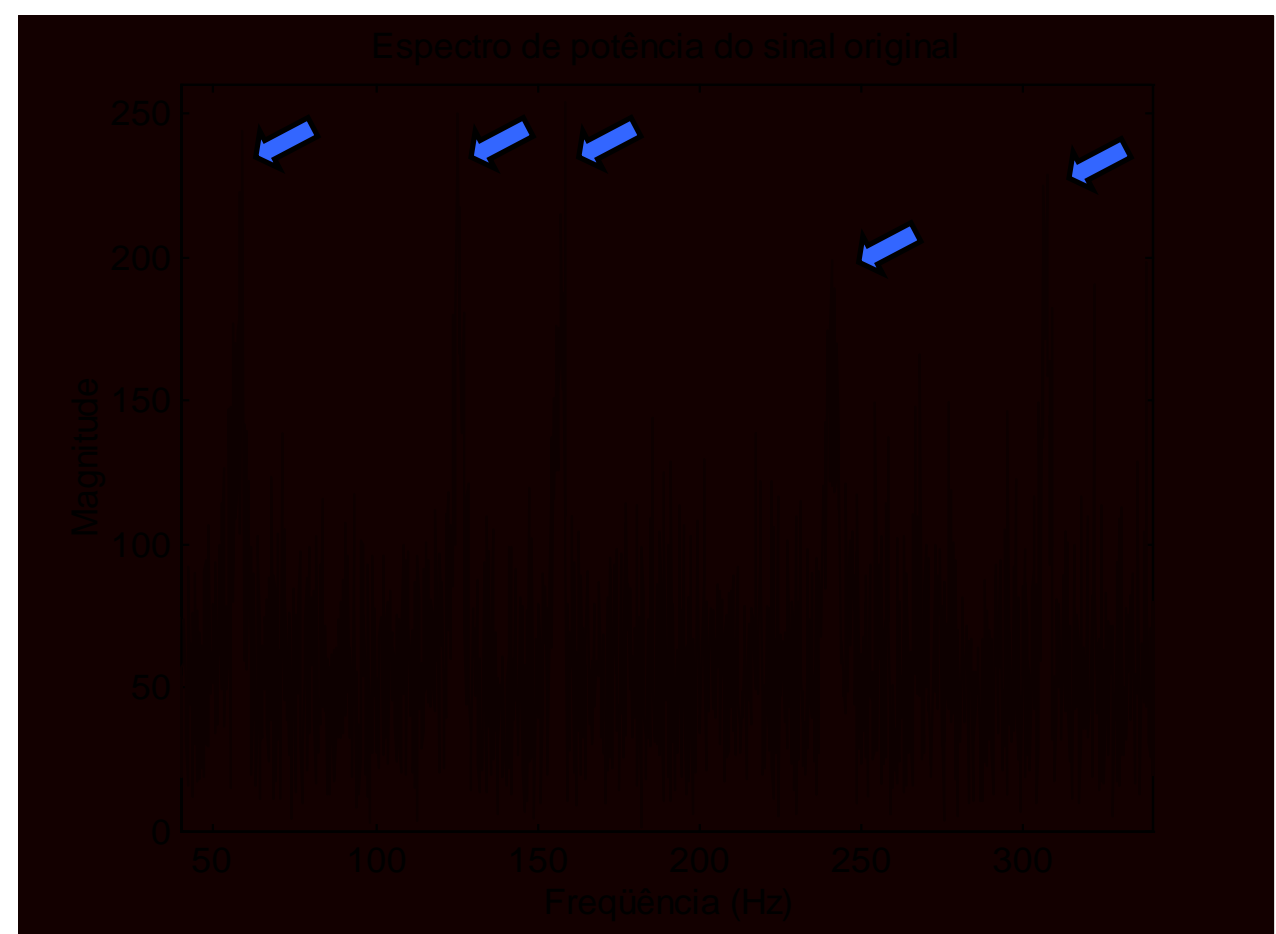

Figura 5. Localização frequencial e distinção das frequências dos pulsos.

Então, foi usado o espectrograma do sinal para definir a localização temporal, utilizando-se outros parâmetros, de forma a aumentar sua resolução temporal. Estes parâmetros foram: 64 pontos para FFT, janela retangular de 64 pontos e sem sobreposição. Este espectrograma pode ser visualizado na figura 6 .

Desta forma, utilizando o recurso de "zoom", foram obtidos aproximadamente os instantes inicial e final dos pulsos, mostrados na tabela 1.

Tabela 1. Instantes inicial e final dos pulsos e sua duração em segundos.

\begin{tabular}{|c|c|c|c|c|}
\hline Pulso & Freqüência (Hz) & Início (s) & Fim (s) & Duração (s) \\
\hline 1 & 58 & 3.185 & 3.440 & 0.255 \\
\hline 2 & 125 & 0.175 & 0.432 & 0.257 \\
\hline 3 & 157 & 2.512 & 2.768 & 0.256 \\
\hline 4 & 241 & 3.024 & 3.280 & 0.256 \\
\hline 5 & 307 & 3.504 & 3.760 & 0.256 \\
\hline
\end{tabular}

Entretanto, como a leitura dos tempos no gráfico foi obtida na transição das cores, a duração obtida acima é um pouco maior do que a real (este acréscimo corresponde aproximadamente à resolução temporal do espectrograma). Portanto, podemos dizer que a duração de todos os pulsos é de aproximadamente $0,25 \mathrm{~s}$. 


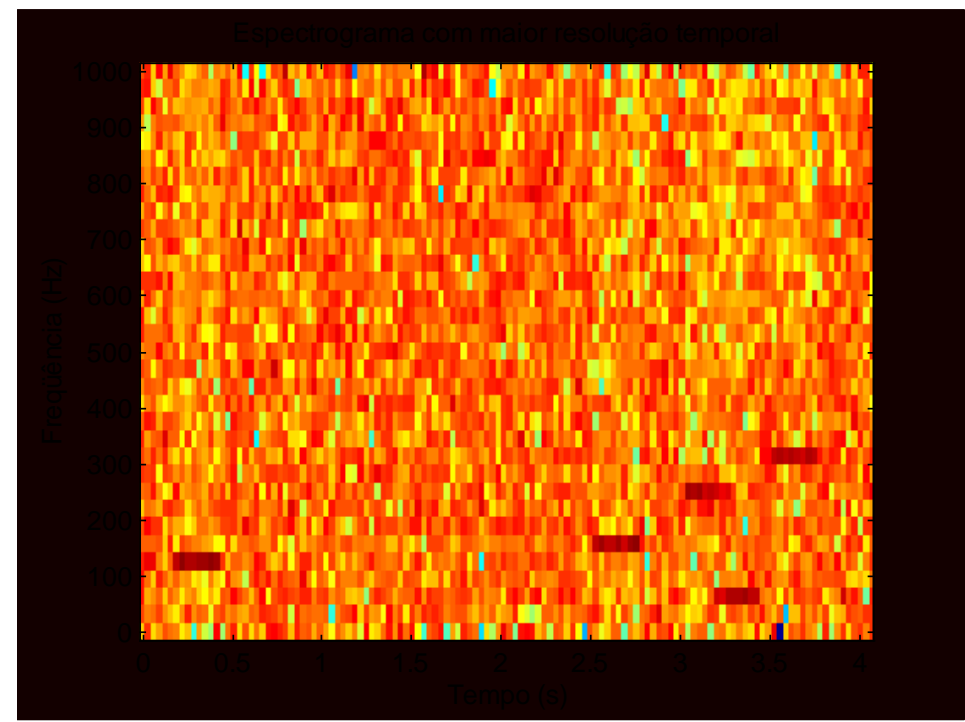

Figura 6. Espectrograma do sinal com maior resolução temporal.

Após a obtenção da localização temporal dos pulsos, cada componente do sinal (correspondente às frequências dos pulsos) obtida anteriormente pôde ser "filtrada" no tempo de modo a anular sua amplitude fora da faixa de duração do pulso. Cada componente do sinal (em verde) e os pulsos obtidos nesta filtragem (em vermelho) são mostrados nas figuras 7, 8, 9, 10 e 11 .

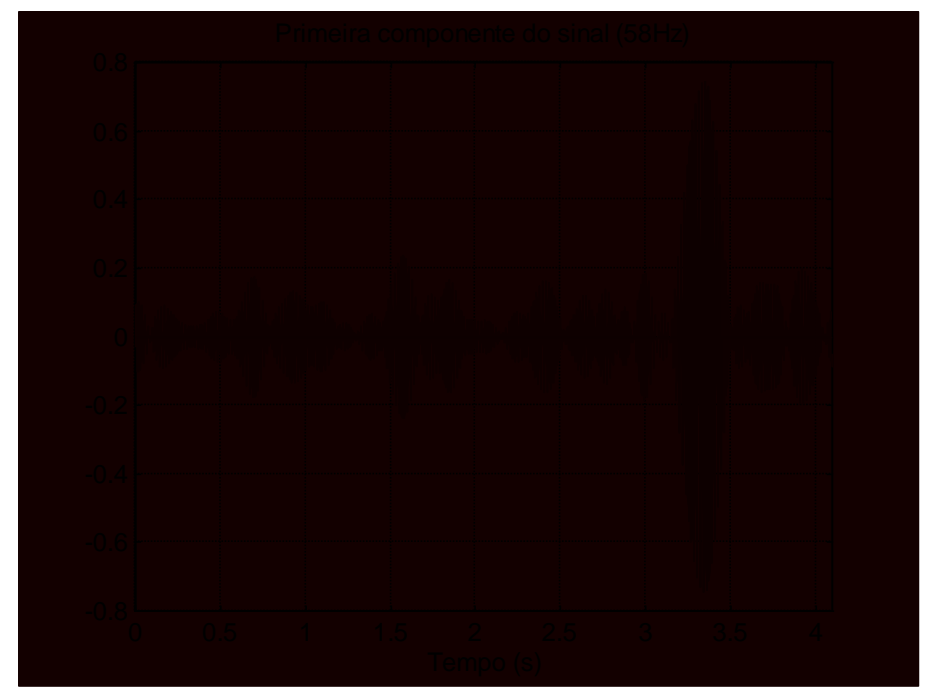

Figura 7. Primeira componente do sinal em aproximadamente $58 \mathrm{~Hz}$

Somando-se todas as componentes filtradas do sinal obtemos uma aproximação do sinal original sem ruídos. Este sinal é mostrado na figura 12. 


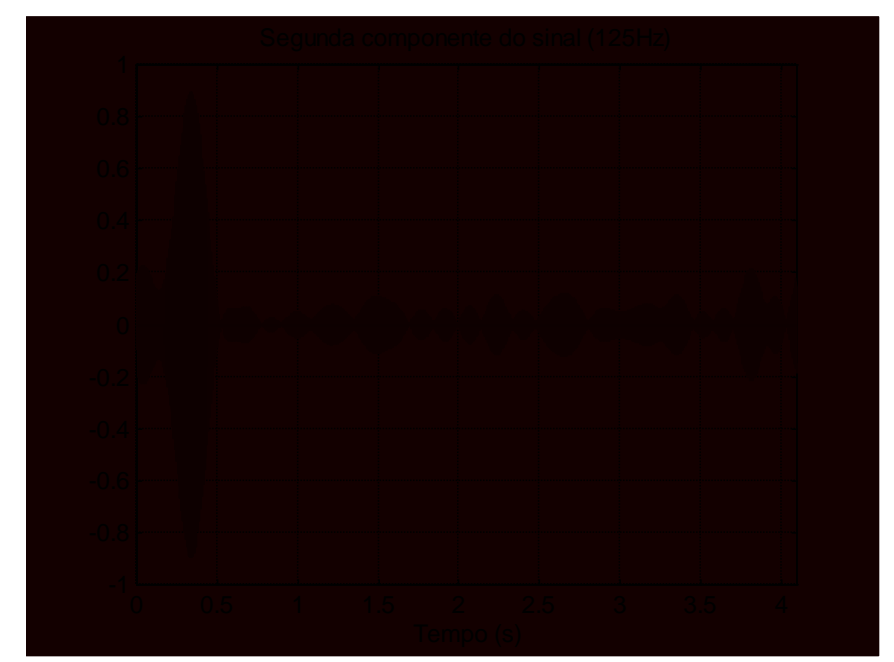

Figura 8. Segunda componente do sinal em aproximadamente $125 \mathrm{~Hz}$

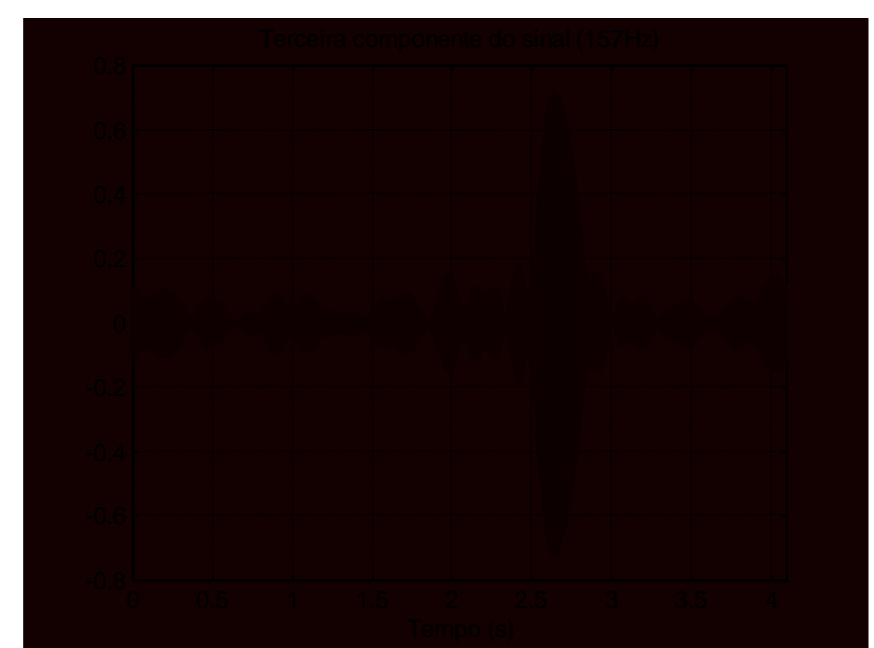

Figura 9: Terceira componente do sinal em aproximadamente $157 \mathrm{~Hz}$

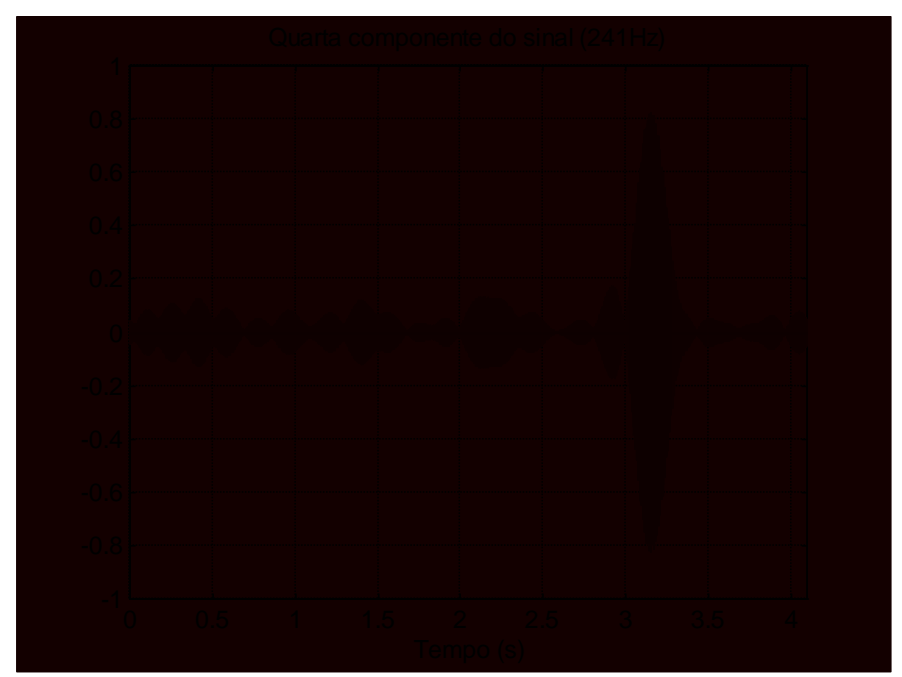

Figura 10. Quarta componente do sinal em aproximadamente $241 \mathrm{~Hz}$ 


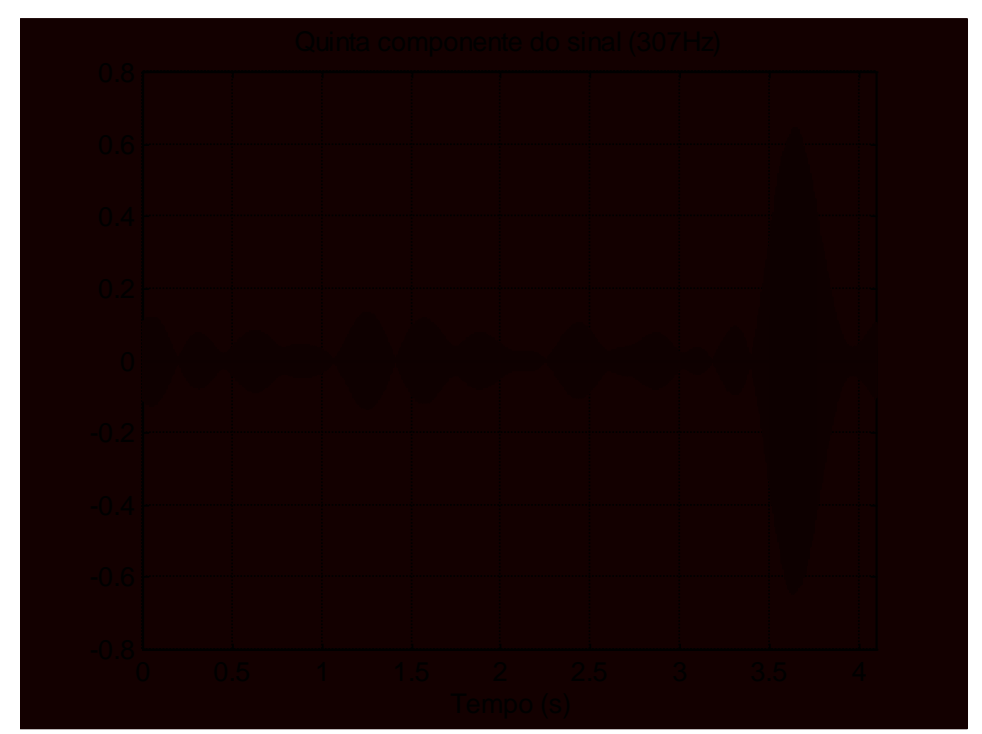

Figura 11. Quinta componente do sinal em aproximadamente $307 \mathrm{~Hz}$

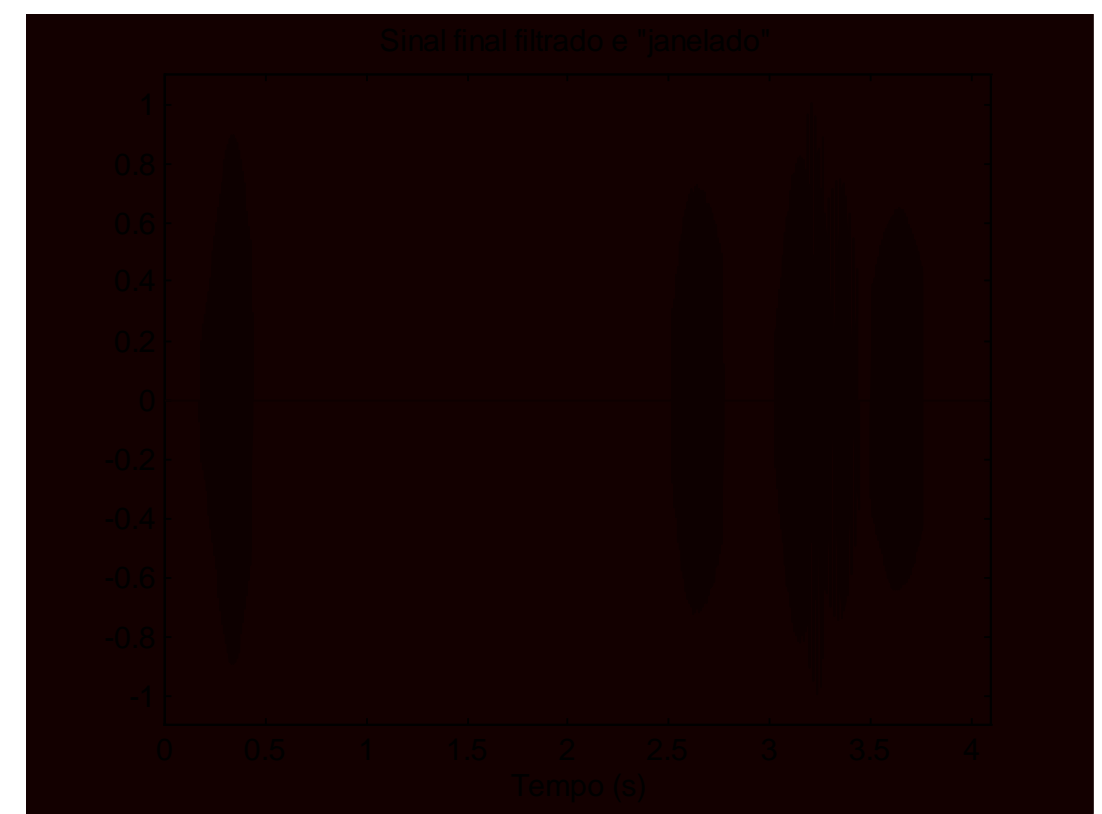

Figura 12. Sinal filtrado: soma das componentes "janeladas" (janela retangular).

\section{CONCLUSÃO}

Esse trabalho apresentou uma análise matemática dos sinais de vibrações causadas pela implosão de bolhas de vapor d'agua presentes no escoamento em grandes dutos de água.

Através de análise dos sinais provenientes do original e aplicando-se técnicas de processamento de sinais, o sinal foi filtrado de seus ruídos.

$$
\text { Concluímos pelos resultados que, }
$$
somando todas as componentes filtradas do sinal, obtemos uma aproximação do sinal original sem ruídos.

\section{REFERÊNCIAS}

SMITH, S. W. 1997. The Scientist and engineer's guide to digital signal processing. 
San Diego, CA: California Technical Publishing, 1997.

DURU, N.; DURU, T.; ABUT, N. Fuzzy logic based noise reduction of digitally recorded speech signal. In: ACM SYMPOSIUM ON APPLIED COMPUTING (SAC '98).

PROCEEDINGS... New York, NY: ACM, 1998.

p.287-29.

https://doi.org/10.1145/330560.330707 